\title{
Erosion patterns in a sediment layer
}

\author{
Adrian Daerr * Peter Lee ${ }^{\dagger}$ José Lanuza, and Éric Clément $\$$ \\ Laboratoire des Milieux Désordonnés et \\ Hétérogènes, UMR7603 - Université Pierre et Marie Curie - Boîte 86, \\ 4 place Jussieu, 75252 Paris CEDEX 05, France
}

(Dated: October 27, 2018)

\begin{abstract}
We report here on a laboratory-scale experiment which reproduces a rich variety of natural patterns with few control parameters. In particular, we focus on intriguing rhomboid structures often found on sandy shores and flats. We show that the standard views based on water surface waves do not explain the phenomenon and we evidence a new mechanism based on a mud avalanche instability.
\end{abstract}

Many patterns observed 1, 2] in natural environments stem from erosion/deposition processes. These structures are related to transport of solid granular particles via a fluid phase that can be either a gas, a liquid, or even a flowing granular phase. They span a huge variety of spatial and temporal scales. Examples of these are fractal river basins [2], meandering rivers 3], dune fields [4], granular avalanches 5], ripple marks [6] on sand banks or on coastal continental platforms. Due in part to its economical and environmental impact, elementary transport processes involved in erosion are still the focus of intense scientific scrutiny.

It is notoriously difficult to provide a fully consistent description of particle laden flows either from a one phase or a two phase point of view [7]. Most of the practical knowledge on erosion comes from empirical laws often derived from field measurements [8]. Several tentatives were made recently to tackle from the statistical physics point of view the dynamics of formation of river basins 2. but many question are raised when one attempts to relate basic transport properties to large scale pattern forming instabilities.

In this letter we report on an experimental setup which is designed to produce a generic situation of a falling water level on an erodible sediment layer. This occurs naturally when the sea retreats from the shore or when a reservoir is drained [1]. We use a plexiglass container with a flat bottom. A square $130 \mathrm{~mm} \times 130 \mathrm{~mm}$ plate of depolished plexiglass slides along the bottom, and is pulled by a motor through a translation stage. The whole setup can be tilted to an arbitrary angle $\theta$. The first step of the experiment is the deposition under water of a sedimented powder layer covering the mobile plexiglass sheet. To this end, the inclination of the whole setup is lowered so that the bottom of the container and the sheet are horizontal $(\theta=0)$. A suspension of alumine oxide powder is then quickly poured into the container. We use commercial

*Electronic address: daerr@ccr.jussieu.fr permanent address: MSC/Univ. Paris VII/PMMH-ESPCI, 10 Rue Vauquelin, F-75231 Paris cedex 05

${ }^{\dagger}$ Electronic address: lee@phys.leidenuniv.nl Department of Physics, Leiden University, Niels Bohrweg 2, NL-2333 RA Leiden

${ }^{\ddagger}$ Electronic address: erc@ccr.jussieu.fr abrasive powder made of rough grains with mean diameter $d \simeq 30 \mu \mathrm{m}$ and density $\rho \simeq 2.75 \mathrm{~g} / \mathrm{cm}^{3}$. The liquid is demineralized water, to which we add hydrochloric acid so as to lower the $p H$ of the suspension to about 4 in order to prevent flocculation of the grains.

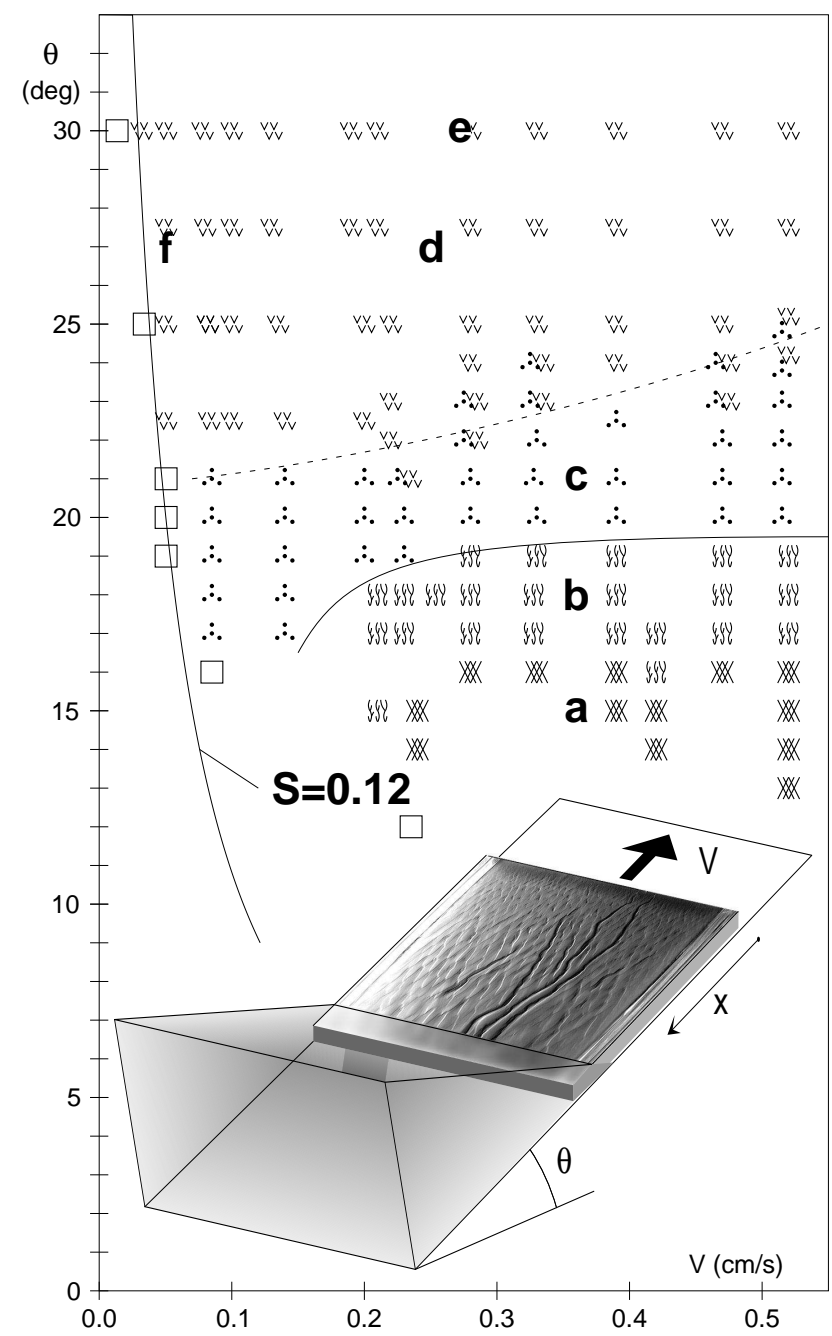

FIG. 1: Phase diagram localizing the different patterns observed in the erosion experiments in the tilt angle $\theta$ - velocity $V$ space. Lines delimiting the domain boundaries are mere guides to the eyes. Letters and symbols correspond to Fig. 2

After deposition of a sedimentary layer of typical 

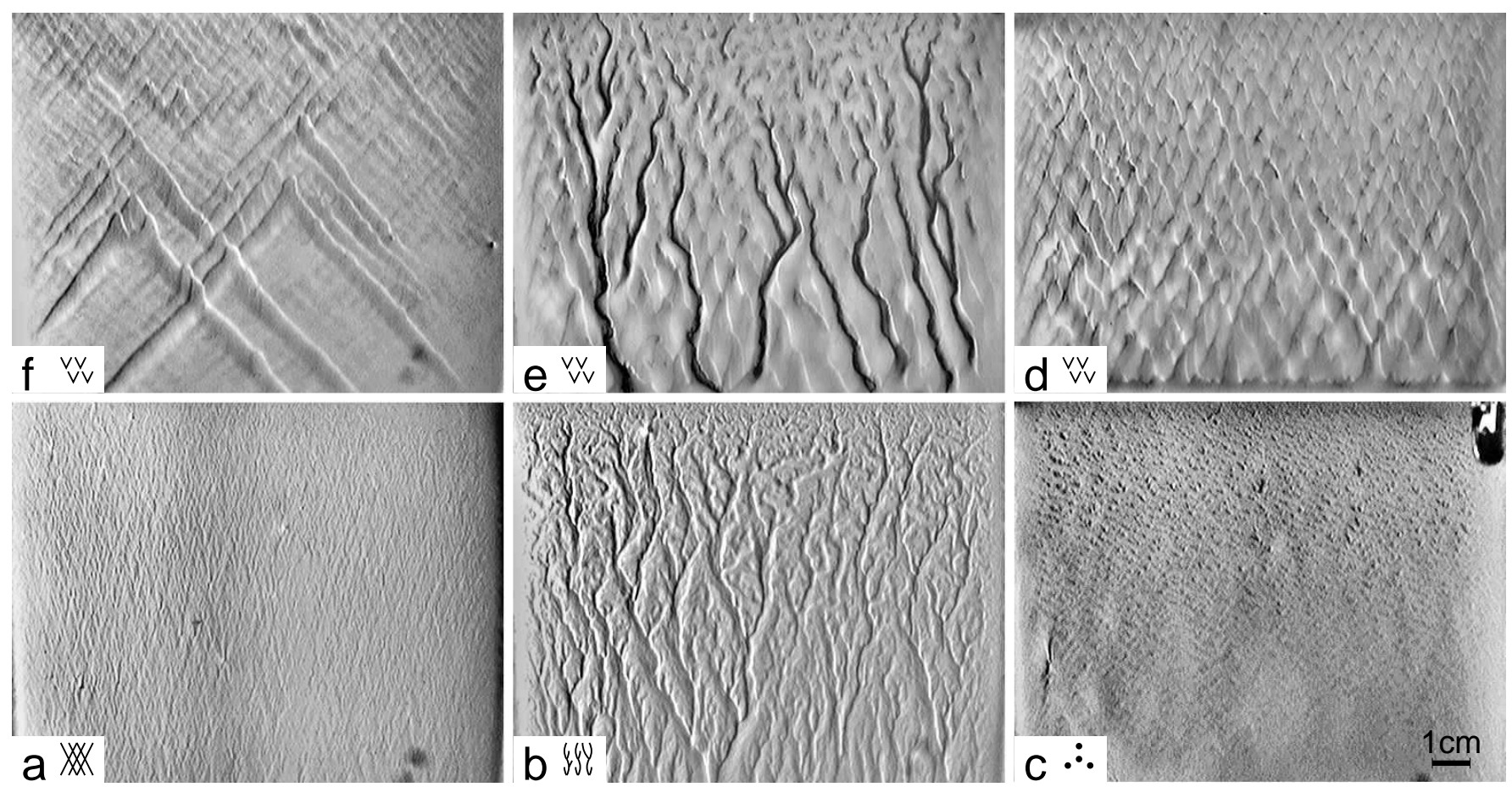

FIG. 2: Patterns observed in the erosion experiment: a crossed hatched pattern, $\mathbf{b}$ disordered branched pattern, $\mathbf{c}$ orange skin, d chevron structure, e chevrons with oblique channels, f localized pulses at chevron onset. The layer appears darker where it has been eroded because the bottom plate is black. A light source to the left creates additional shading.

height $1 \mathrm{~mm}$, the container and the plate are tilted to an angle $\theta$. Part of the liquid is then slowly drained, until the contact line of the liquid free surface with the tilted bottom of the container reaches about $1 \mathrm{~cm}$ below the top of the mobile sheet. Then the sliding plate is pulled out of the liquid at a speed $V$ (see inset of Fig. 1). The layer is filmed by a CCD camera. Its position is fixed with respect to the plate and its optical axis is perpendicular to the surface. This simple set-up allows us to observe a variety of phenomena and structures depending on two control parameters, the angle $\theta$ and the speed $V$. The "phase diagram" is sketched on Fig. [1 When the sedimented granular layer is pulled out very slowly $(V \leq 0.04 \mathrm{~cm} / \mathrm{s})$, no pattern is observed. The liquid seeps out of the sediment, which dries progressively without being altered. However, above a critical speed and tilt corresponding on Fig. 1 to empty squares, we observe the formation of erosive patterns on the granular sediment surface. For decreasing angle, the patterns faint away progressively and for a tilt angle $\theta<13^{\circ}$, it is often difficult to witness of their presence.

At velocities greater than approx. $0.1 \mathrm{~cm} / \mathrm{s}$, surface structures appear clearly when the layer is tilted to more than about $14^{\circ}$. For angles close to this value, the draining liquid leaves a cross-hatched, dense pattern of very small and shallow channels (see Fig. 27).

Around $18^{\circ}$, we obtain a branched, disordered rivernetwork, whose biggest branches have widths of about $1 \mathrm{~mm} \approx 30 d$ (Fig. $2 \mathrm{~b}$ ). After the passage of the water contact line, the surface of the sediment is still smooth and the pattern appears with a delay of few tens of seconds. The dynamical evolution of the structure can last as long as two minutes. First, small localized structures with a characteristic angle similar to the previous cross-hatched pattern appear almost everywhere and then, bigger and bigger disordered structures are created as they merge under the action of erosion and sediment transport. We will call this regime the disordered regime, refering to the random aspect of the final network. Transition between cross-hatched and branched is progressive.

For velocities higher than $0.1 \mathrm{~cm} / \mathrm{s}$ and slopes increased above approximately $19^{\circ}$, there is a sharp $\left(1-2^{\circ}\right)$ transition to a regime of dimples with a structural aspect similar to an orange skin (see Fig. 22). For steeper slopes, we observe the progressive onset of a chevron pattern characterized by a well defined angle (see Fig. 22 d). The crossover region is indicated approximatively by a dashed line on the phase diagram. The chevron pattern forms quickly (typically five seconds) behind the receding liquid contact line. The rhomboid elements characterizing this structure have a slightly rounded downhill tip and a height profile like fish scales or roof tiles, i.e. the sediment is thickest at the downhill tip, with a shallow decrease uphill, and a sharp lower edge.

Finally, this regime is limited by the maximal stability angle $\theta_{m}=35^{\circ}$ above which the sediment layer would spontaneously avalanche as a whole.

From the present experiment, we observe no systematic variation of the chevron wavelength $\lambda$ with velocity or tilt angle. Nevertheless, the spacing of the chevrons 
has a tendency to grow as the contact line recedes with a mechanism akin to defect fusion; we obtain a mean spacing $\lambda=5 \mathrm{~mm} \pm 2 \mathrm{~mm}$. On the other hand, systematic experiments at constant angle $\theta=30^{\circ}$, show clearly a decrease of the chevron pattern opening angle $\varphi$ from $90^{\circ}$ to $30^{\circ}$ for increasing velocity (see Fig. 3).

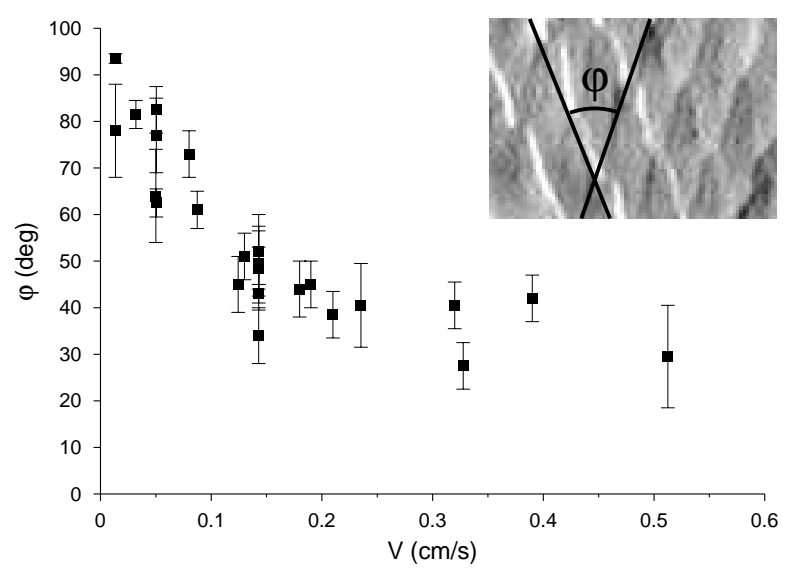

FIG. 3: Chevron alignment angle as a function of velocity. Error bars indicate measurement variations

Various rhomboid patterns have already been described by geologists in natural environments [1] like on sea-ward facing beach slopes [9], in reservoirs or river beds after a full drainage of the water. Although there seem to be several distinct types, only few attempts at explaining a possible mechanism were made [9]. Previous observations and the few experiments available commonly attribute the formation of chevron patterns to instabilities at the free surface of the flowing water layer (such as hydraulic jumps) which couple to the bottom profile [10]. Although this could be true for certain types of rhomboid ripples in shallow, fast flowing rivers, it cannot be the case for the chevron regime described here. Indeed, the largest estimation for the Froude number we can make at the chevron onset is $\mathrm{Fr}=V / \sqrt{g d}=5 \cdot 10^{-2}$, which certainly rules out factors like hydraulic jumps of the water layer.

Now we seek to clarify the physical conditions associated with the onset of pattern formation. An estimation of the Darcy flow velocity $V_{D}$ inside the powder yields $V_{D}=g K / \nu \simeq 10^{-5} \mathrm{~m} / \mathrm{s}$, which is much smaller than the retrieval velocity $V$. This $V_{D}$ value is obtained with a permeability $K=10^{-12} \mathrm{~m}^{2}$ obtained experimentally, and a kinematic viscosity $\nu=10^{-6} \mathrm{~m}^{2} / \mathrm{s}$. Moreover, the capillary length corresponding to a $30 \mu \mathrm{m}$ porous medium under gravity forces is about $20 \mathrm{~cm}$. It is thus legitimate to consider that the sediment remains fully soaked with water during retrieval of the plate. To estimate the shear exerted by the liquid film at the surface, we calculate its thickness $h(x, t)$ in the plate reference frame. The retrieval of the plate begins at $t=0$, and $x=0$ is the initial position of the contact line between liquid surface and sediment. Assuming that the flow is viscous, so that the average local flow velocity is given by $\bar{V}(x, t)=g \sin \theta h(x, t)^{2} / 3 \nu$, the
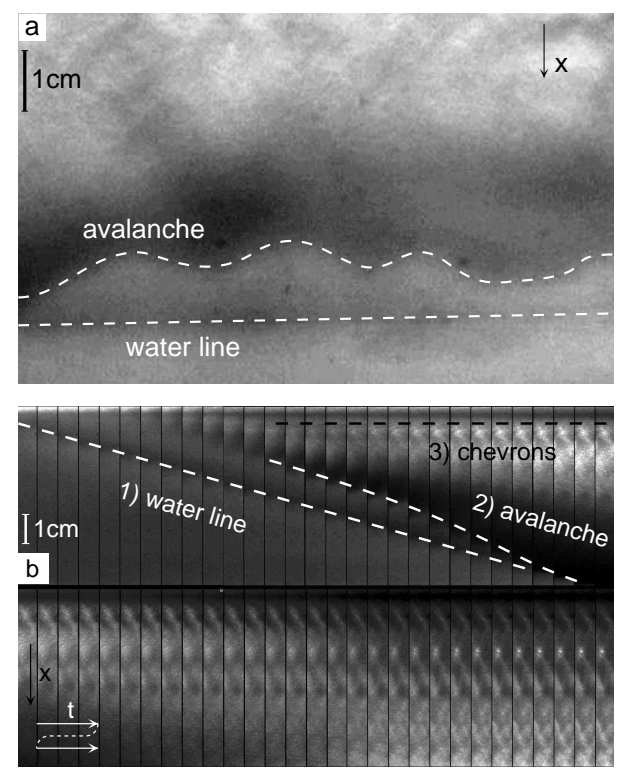

FIG. 4: Dynamics of the chevron formation. a snapshot of the mud avalanche above the contact line. b spatio-temporal diagram (split across two lines) showing how chevrons appear in the wake of an avalanche.

mass conservation equation $\partial_{t} h+\partial_{x}(h(x, t) \bar{V}(x, t))=0$ yields, for small free flow slopes, a self consistent solution: $h(x, t)=\sqrt{2 \nu x / \operatorname{tg} \sin \theta}$. Note that we neglected both capillary and hydrostatic pressure terms because of the small thickness and curvature of the flowing layer. This approximation ceases to be valid close to the origin and in the vicinity of the junction with the flat water level. Note also that the contact line cannot move within this approximation, which corresponds to a situation of total wetting on the sediment. The maximum height, just above the reservoir water level, is thus evaluated to be $h=\sqrt{(\nu / g \sin \theta) V}=10 \mu \mathrm{m} \approx d$. The first conclusion is that the Reynolds number, $\operatorname{Re}=h V / \nu=0.03$, is small enough to justify the lubrication approximation. Second, the ratio of the shear exerted by the fluid on a grain at the bottom and its apparent weight yields a common criterion for the onset of erosion called Shield's number,

$$
S=\frac{\rho_{w} \tan \theta}{\Delta \rho} \frac{h(x, t)}{d} \simeq\left(\frac{V}{V_{0}}\right)^{1 / 2} \text { with } V_{0}=\left(\frac{\Delta \rho}{\rho_{w}}\right)^{2} \frac{g d^{2}}{\nu}
$$

$\rho_{w}$ is the density of water and $\Delta \rho=\rho-\rho_{w}$ the density contrast between grains and liquid. On Fig. 2 we plotted the $V(\theta)$ curve corresponding $S=0.12$. The scaling implications of this formula should be put to test more systematically but so far, it seems to reproduce remarkably the shape of the limit were erosion patterns are evidenced. Note that a Shields number of value $S=0.12$ is marginally large to represent a situation where a grain would be spontaneously dislodged under the action of viscous shear. On the other hand, when interpreted in the frame work of a Coulomb criterion for the sediment layer stability, the shearing strength due to viscous 
forces could be large enough to trigger an avalanche of wet grains. This is indeed what we observe at an angle above around $18^{\circ} \pm 1^{\circ}$. For this purpose, we visualized the experiment in the chevron regime by lighting the flow through the bottom of the set-up (Fig. 4a): on this figure, darker spots corresponding to thicker sediment layers. On Fig. «b we present a space-time view of the dynamical process for erosion and chevron formation. Each stripe of actual size $0.7 \times 6.1 \mathrm{~cm}^{2}$ corresponds to the central part of a picture like 4a. Each successive stripe corresponds to a time lag of $0.04 \mathrm{~s}$. The line labeled by 1 indicates the water line retreating at constant velocity. The line by labeled 2 shows the front of a sediment avalanche triggered at the very top of the soaked layer. Clearly this avalanche accelerates as grains accumulate at the front, and it eventually catches up with the waterline. As the avalanche proceeds, a spatially modulated pattern of settling sediment occurs in the wake of the surge (line 3 indicates first chevrons) from the top downwards. During this settlement, an horizontal structure is created with a well defined spatial selection already visible in the vicinity of the avalanche onset. This original structure will give rise to the chevrons pattern since the subsequent sedimented structure will occur further down with an horizontal spatial shift of roughly one half of the wave length. Then a similar scenario is taking place for the next structures down and so on. After final settlement of a chevron line, sediment will still flow but only between adjacent chevrons. Now, instead of an avalanche flow, we rather have a channel flow which displays an erosive activity sharpening the pattern until it reaches its final form. This final erosion process appears as a backwards wave. At larger velocities the channel flow is sometimes able to erode the layer down to the bottom of the plate, producing a river-like network as in Fig. 22.

There are many open questions left on why such a structure is likely to occur in the wake of the avalanche, and why it has such a well defined wave-length. Here we mention two plausibly relevant paths of thought. Recent experiments on sheared dense suspensions in a rotating drum show that a particle dense flow is instable under shear [1]. Particle segregation occurs in the transverse direction, producing regions denser in solid particles that may enhance in our situation a localized sedimentary process. Note also that the dynamical aspects related to this mud avalanche bears many similarities with studies on instabilities and pattern formation in falling viscous sheets [12]. To our understanding, this chevron regime reveals an interesting and open problem of mud flow and sedimentation wave propagation. Along this line, it is interesting to note that just at the onset of instability, in a tiny velocity region, a regime of localized pulses crossing each other at $90^{\circ}$ is observed (see Fig. 22), which is actually strongly reminiscent of a non-linear waves phenomenology.

In conclusion, we presented new results on a laboratory scale experiment producing a rich variety of patterns due to erosion deposition processes. The notion of "phase" we use in the scope of this manuscript is qualitative (based on visual distinction), and should be defined more precisely in terms of an order parameter. We show that contrarily to previous propositions these structures do not occur as a consequence of water surface waves, but we postulate that they are triggered by water viscous strain in low Reynolds and Froude numbers regime. The corresponding Shield's criterion implies certain scalings of the onset with grain size, viscosity etc., which are currently investigated. We especially focussed here on an intriguing rhomboid-like (chevron) structure organized with a typical distance and a coherent angle between chevrons. This avalanche regime is clearly separated from another erosion mechanism producing disordered networks of branched or cross-hatched channels. In the chevron regime we evidence a new mechanism based on the triggering of a dense sediment avalanche. The observed patterns stems from instabilities initiated in the wake of the avalanche. Erosion/deposition mechanisms further develop in order to carve the pattern to its final chevron form.

Acknowledgments. We thank P. Gondret, F. Métivier and J. Socolar for interesting discussions, J. Treiner for photographs of natural erosion patterns. The help of G. Guéna on the experiment is also acknowledged.
[1] J. L. R. Allen, Developments in Sedimentology: Sedimentary Structures (Elsevier (Amsterdam, NL), 1984).

[2] P. S. Dodds and D. H. Rothman, Annu. Rev. Earth Planet. Sci. 28, 571 (2000).

[3] B. F. Edwards and D. H. Smith, Phys. Rev. E 65 (2002).

[4] K. Kroy, G. Sauermann, and H. J. Herrmann, Phys. Rev. Lett. 88 (2002).

[5] A. Daerr, Phys. Fluids 13, 2115 (2001).

[6] A. Betat, V. Frette, and I. Rehberg, Phys. Rev. Lett. 83, 88 (1999).

[7] H. J. Herrmann, J.-P. Hovi, and S. Luding, eds., Physics of Dry Granular Media, vol. 350 of NATO ASI Series
$E$ (Kluwer (Dordrecht, NL), 1998), proceedings of the Summer 1997 Conference in Cargese, France.

[8] W. H. Graf, Hydraulique Fluviale, vol. 16 of Traité de Génie Civil de l'EPF de Lausanne (Presses Polytechniques et Universitaires Romandes (Lausanne, $\mathrm{CH}$ ), 1993).

[9] Stauffer1976 hoyt1963 demarest1947 woodford1935.

[10] Karcz1980 chang1970.

[11] M. Tirumkudulu et al., Phys. Fluids 11, 507 (1999).

[12] J. Liu, J. B. Schneider, and J. P. Gollub, Phys. Fluids 7, 55 (1995). 